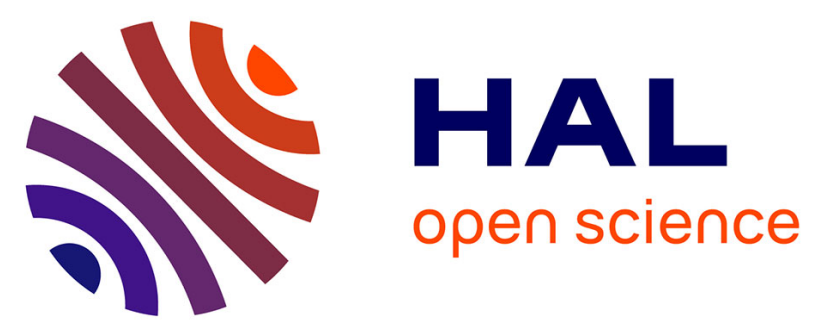

\title{
DEEP EXPECTATION-MAXIMIZATION FOR IMAGE RECONSTRUCTION FROM UNDER-SAMPLED POISSON DATA
}

Antonio Lorente Mur, Paul Bataille, F Peyrin, Nicolas Ducros

\section{- To cite this version:}

Antonio Lorente Mur, Paul Bataille, F Peyrin, Nicolas Ducros. DEEP EXPECTATIONMAXIMIZATION FOR IMAGE RECONSTRUCTION FROM UNDER-SAMPLED POISSON DATA. 2021 IEEE 18th International Symposium on Biomedical Imaging (ISBI), Apr 2021, Nice, France. pp.1535-1539, 10.1109/ISBI48211.2021.9433805 . hal-02944869v2

\section{HAL Id: hal-02944869 \\ https://hal.science/hal-02944869v2}

Submitted on 15 Nov 2021

HAL is a multi-disciplinary open access archive for the deposit and dissemination of scientific research documents, whether they are published or not. The documents may come from teaching and research institutions in France or abroad, or from public or private research centers.
L'archive ouverte pluridisciplinaire $\mathbf{H A L}$, est destinée au dépôt et à la diffusion de documents scientifiques de niveau recherche, publiés ou non, émanant des établissements d'enseignement et de recherche français ou étrangers, des laboratoires publics ou privés. 


\title{
DEEP EXPECTATION-MAXIMIZATION FOR IMAGE RECONSTRUCTION FROM UNDER-SAMPLED POISSON DATA
}

\author{
Antonio Lorente Mur, Paul Bataille, Françoise Peyrin, Nicolas Ducros \\ Univ Lyon, INSA-Lyon, UCB Lyon 1,CNRS, Inserm, CREATIS UMR 5220, U1206, Lyon, France
}

\begin{abstract}
Many biomedical imaging techniques, such as computerized tomography, positron emission tomography, and optical microscopy, involve reconstruction of an image from a sequence of a few linear measurements that are corrupted by Poisson noise. In this study, we focus on computational optics, and more precisely single-pixel imaging, where the set-up acquires some of the coefficients of the Hadamard transform of the image of the scene.

Recently, this problem has benefited from the advent of deep learning. Although deep methods were initially considered as black boxes, they are now understood as learnable optimisation schemes. Here, we propose a network architecture based on the expectation-maximization algorithm that optimizes the maximum a posteriori of the unknown image for measurements corrupted by Poisson noise. This leads to an interpretable network that generalizes several existing approaches.

Finally, we present some reconstruction results from simulated data and from experimental acquisitions from a singlepixel camera. Our network yields higher reconstruction peak signal-to-noise ratios than other similar approaches.
\end{abstract}

Index Terms - Image reconstruction, deep learning, expectation-maximization, model-based optimization methods, single-pixel imaging.

\section{INTRODUCTION}

Single-pixel imaging is an extreme configuration of computational optics, where a single point detector is used to recover a two-dimensional image [1]. Since the original work of Duarte and coworkers [2], single-pixel imaging has been successfully applied to fluorescence microscopy [3], hyperspectral imaging [4], image-guided surgery [5], fluorescence lifetime imaging [6], and others. A single-pixel camera measures the dot products between an image and a set of two-dimensional functions that are implemented through a spatial light modulator. To limit acquisition times, it is highly desirable to reduce the number of light patterns and acquisition time, which leads to an under-determined inverse problem with Poisson noise.
Image reconstruction from noisy measurements where the number of unknowns is larger than the number of measurements is a generic problem that has several applications in computational imaging. Such problems have long benefited from the theory of compressed sensing, but recent advances in deep learning have revolutionized the field [7]. In particular, convolutional neural networks have shown great success for solving inverse problems $[8,9]$.

Traditional optimisation-based approaches for image reconstruction have inspired deep-learning-based reconstructors to solve inverse problems. For instance, they have been used as priors for manifolds of natural images [10], and as projectors for sets of natural images [11]. While these methods have outperformed traditional image reconstruction methods, they implicitly assume signal-independent noise, and are therefore not optimal for tackling problems with Poisson noise.

Inspired by the unrolled deep-learning methods, such as indicated by [10], we derive an iterative neural network based on the expectation-maximization (EM) algorithm [12], to specifically tackle data corrupted by noise with nonconstant variance. This provides a framework for handling signaldependent noise flowing through iterations, which generalizes existing methods.

In Section 2, we model a compressive acquisition device and describe the associated reconstruction problem. In Section 3, we introduce the core ideas for our Bayesian inversion approach to the inverse problem. In Section 4, we propose our EM-based unrolled network to process data corrupted by Poisson noise. In Section 5, we describe how we implement and train the network. In Section 6, we report and analyze our reconstruction results, before concluding in Section 7.

\section{COMPRESSIVE IMAGING}

Let $f \in[0,1]^{N}$ be the image to acquire. The main idea of compressive optics is to measure $\boldsymbol{m}=\boldsymbol{H}_{1} \boldsymbol{f}$ using hardware, and to recover $f$ using software. The system matrix $\boldsymbol{H}_{1} \in \mathbb{R}^{M \times N}$, with $M<N$, collects the patterns that are sequentially uploaded on a spatial light modulator to get the measurement vector. The patterns are traditionally chosen from within a basis $\boldsymbol{H} \in \mathbb{R}^{N \times N}$, i.e., $\boldsymbol{H}_{1}=\boldsymbol{S H}$ with 
$\boldsymbol{S}=\left[\boldsymbol{I}_{M}, \mathbf{0}\right]$. Classical choices include Fourier, discrete cosine, wavelets, and Hadamard bases [13].

The acquisition, which is corrupted by Poisson noise [14], can be modeled as

$$
\begin{gathered}
\mathbf{m}^{\alpha}=\left(\hat{\mathbf{m}}_{+}^{\alpha}-\hat{\mathbf{m}}_{-}^{\alpha}\right) / \alpha \\
\hat{\mathbf{m}}_{+}^{\alpha} \sim \mathcal{P}\left(\alpha \boldsymbol{H}_{1}^{+} \boldsymbol{f}\right) \quad \text { and } \quad \hat{\mathbf{m}}_{-}^{\alpha} \sim \mathcal{P}\left(\alpha \boldsymbol{H}_{1}^{-} \boldsymbol{f}\right) .
\end{gathered}
$$

where $\alpha$ is the image intensity (in photons), and $\mathbf{m}^{\alpha}$ is the normalized noisy measurement vector. Note that the implementation of the negative values of $\boldsymbol{H}_{1}$ is carried out through the use of positive patterns $\boldsymbol{H}_{1}^{+}$and $\boldsymbol{H}_{1}^{-}$, such that $\left(\boldsymbol{H}_{1}^{+}\right)_{i, n}=\max \left(\left(\boldsymbol{H}_{1}\right)_{i, n}, 0\right)$ and $\left(\boldsymbol{H}_{1}^{+}\right)_{i, n}=$ $\max \left(-\left(\boldsymbol{H}_{1}\right)_{i, n}, 0\right)$ (see [15] for details).

\section{BAYESIAN RECONSTRUCTION}

Bayesian inversion aims at the computing of a point-wise estimator of the probability density function of $(\mathbf{f} \mid \mathbf{m})$. Assuming that $\mathbf{f}$ has finite mean and variance, the conditional expectation is given by the minimum mean squared error

$$
\boldsymbol{f}^{*}=\mathbb{E}\left(\mathbf{f} \mid \mathbf{m}^{\alpha}=\boldsymbol{m}^{\alpha}\right)=\mathcal{G}^{*}\left(\boldsymbol{m}^{\alpha}\right),
$$

where the mapping $\mathcal{G}^{*}$ is given by

$$
\mathcal{G}^{*} \in \underset{\mathcal{G}}{\operatorname{argmin}} \mathbb{E}\left(\left\|\mathcal{G}\left(\mathbf{m}^{\alpha}\right)-\mathbf{f}\right\|^{2}\right) .
$$

For general distributions of $\mathbf{f}$, the mapping $\mathcal{G}^{*}$ is nonlinear, and there are no closed-form solutions.

Instead of solving Equation (3), which is computationally intractable in general, deep-learning-based methods replace the expectation by the empirical mean over a database, and optimize a mapping $\mathcal{G}_{\omega}$ within a family of mappings parameterized by some weights $\omega$. The choice of the form of the nonlinear mapping $\mathcal{G}_{\boldsymbol{\omega}}$ is central to the reconstruction. Much attention has been devoted to neural network models where the architecture mimics iterative maximum a posteriori (MAP) optimisation schemes [16].

\section{PROPOSED METHOD}

\subsection{Unrolling the EM algorithm}

We aim to compute the MAP for our problem

$$
\underset{\boldsymbol{f}}{\operatorname{argmax}} \log p\left(\boldsymbol{m}^{\alpha} \mid \boldsymbol{f}\right)+\log p(\boldsymbol{f}),
$$

where we assume $p\left(\boldsymbol{m}^{\alpha} \mid \boldsymbol{f}\right) \propto \exp -\frac{1}{2}\left\|\boldsymbol{H}_{1} \boldsymbol{f}-\boldsymbol{m}^{\alpha}\right\|_{\boldsymbol{\Sigma}_{\boldsymbol{\alpha}}^{-1}}^{2}$, and $\boldsymbol{\Sigma}_{\boldsymbol{\alpha}}$ is the covariance of the noise, and $p(\boldsymbol{f})$ is an unknown probability density function.

The EM algorithm has commonly been used to estimate the MAP for image reconstruction tasks [17]. This is an iterative algorithm that produces a sequence of estimations $\left\{\boldsymbol{f}^{(k)}\right\}$ that converge to a local maximum of Equation (4). Every iteration of the EM algorithm is based on two steps.

The expectation step computes the conditional expectation of the log-likelihood of $\mathbf{f}$ with respect to an auxiliary random variable $\mathbf{x}$ (commonly called the complete data), given the current estimate $\boldsymbol{f}^{(k)}$ and the measurements $\boldsymbol{m}^{\alpha}$

$$
\mathcal{Q}\left(\boldsymbol{f} \mid \boldsymbol{f}^{(k)}\right)=\int p\left(\boldsymbol{x} \mid \boldsymbol{m}^{\boldsymbol{\alpha}}, \boldsymbol{f}^{(k)}\right) \log p\left(\boldsymbol{x}, \boldsymbol{m}^{\boldsymbol{\alpha}} \mid \boldsymbol{f}\right) d \boldsymbol{x}+p(\boldsymbol{f})
$$

This quantity is then maximized with respect to $f$ during the maximization step, to produce the next iteration

$$
\boldsymbol{f}^{(k+1)}=\underset{\boldsymbol{f}}{\operatorname{argmax}} \mathcal{Q}\left(\boldsymbol{f} \mid \boldsymbol{f}^{(k)}\right)
$$

The EM algorithm converges to a local maximum of Equation (4) if the complete data $\mathrm{x}$ satisfy some admissibility proprieties. When $p\left(\boldsymbol{m}^{\alpha} \mid \boldsymbol{f}\right)$ is Gaussian, $\mathbf{x}$ can be chosen as a Gaussian vector, such that [12]

$$
\begin{aligned}
\mathbf{x} & \sim \mathcal{N}(\boldsymbol{H} \boldsymbol{f}, \boldsymbol{\Sigma}) \\
\mathbf{m}^{\alpha} \mid \mathbf{x} & \sim \mathcal{N}\left(\boldsymbol{S} \mathbf{x}, \boldsymbol{\Sigma}_{\alpha}\right) .
\end{aligned}
$$

Therefore, $\mathbf{x}$ satisfies the admissibility properties if $\boldsymbol{H}_{1} \tilde{\boldsymbol{\Sigma}} \boldsymbol{H}_{1}^{\top}$ $=\boldsymbol{S} \boldsymbol{\Sigma} \boldsymbol{S}^{\top}$, where $\tilde{\boldsymbol{\Sigma}}$ is the covariance of $\mathbf{f}$, and Equation (5) simplifies to

$$
\begin{aligned}
\overline{\boldsymbol{x}}^{(k)} & =\mathbb{E}\left(\mathbf{x} \mid \boldsymbol{m}^{\boldsymbol{\alpha}}, \boldsymbol{f}^{(k)}\right), \\
\mathcal{Q}\left(\boldsymbol{f} \mid \boldsymbol{f}^{(k)}\right) & =\log p\left(\overline{\boldsymbol{x}}^{(k)} \mid \boldsymbol{f}\right)+\log p(\boldsymbol{f}) .
\end{aligned}
$$

Using classical properties of Gaussian vectors (see Chapter 5 of [18]), we can rewrite these two steps

$$
\begin{aligned}
& \overline{\boldsymbol{x}}^{(k)}=\underset{\boldsymbol{x}}{\operatorname{argmin}}\left\|\boldsymbol{S} \boldsymbol{x}-\boldsymbol{m}^{\alpha}\right\|_{\boldsymbol{\Sigma}_{\boldsymbol{\alpha}}^{-1}}^{2}+\left\|\boldsymbol{x}-\boldsymbol{H} \boldsymbol{f}^{(k)}\right\|_{\boldsymbol{\Sigma}^{-1}}^{2} \\
& \boldsymbol{f}^{(k+1)}=\underset{\boldsymbol{f}}{\operatorname{argmin}}\left\|\overline{\boldsymbol{x}}^{(k)}-\boldsymbol{H} \boldsymbol{f}\right\|_{\boldsymbol{\Sigma}^{-1}}^{2}-\log p(\boldsymbol{f}) .
\end{aligned}
$$

As $p(\boldsymbol{f})$ is an unknown probability density function, we propose to estimate Equation (11b) using a neural network $\mathcal{D}_{\boldsymbol{\omega}}$

$$
\begin{aligned}
& \overline{\boldsymbol{x}}^{(k)}=\underset{\boldsymbol{x}}{\operatorname{argmin}}\left\|\boldsymbol{S} \boldsymbol{x}-\boldsymbol{m}^{\alpha}\right\|_{\boldsymbol{\Sigma}_{\boldsymbol{\alpha}}^{-1}}^{2}+\left\|\boldsymbol{x}-\boldsymbol{H} \boldsymbol{f}^{(k)}\right\|_{\boldsymbol{\Sigma}^{-1}}^{2} \\
& \boldsymbol{f}^{(k+1)}=\mathcal{D}_{\boldsymbol{\omega}}\left(\boldsymbol{H}^{\top} \overline{\boldsymbol{x}}^{(k)}\right) .
\end{aligned}
$$

Note that Equation (12a) is commonly called the dataconsistency layer in the literature on deep unrolled methods [10].

\subsection{Implementation of the data-consistency layer}

Introducing the variable $\boldsymbol{y}^{(k)}=\overline{\boldsymbol{x}}^{(k)}-\boldsymbol{H} \boldsymbol{f}^{(k)}$, the analytical solution of Equation (12a) is given by

$$
\boldsymbol{y}^{(k)}=\left[\begin{array}{c}
\boldsymbol{\Sigma}_{1} \\
\boldsymbol{\Sigma}_{21}
\end{array}\right]\left(\boldsymbol{\Sigma}_{\alpha}+\boldsymbol{\Sigma}_{1}\right)^{-1}\left(\boldsymbol{m}^{\alpha}-\boldsymbol{H}_{1} \boldsymbol{f}^{(k)}\right),
$$




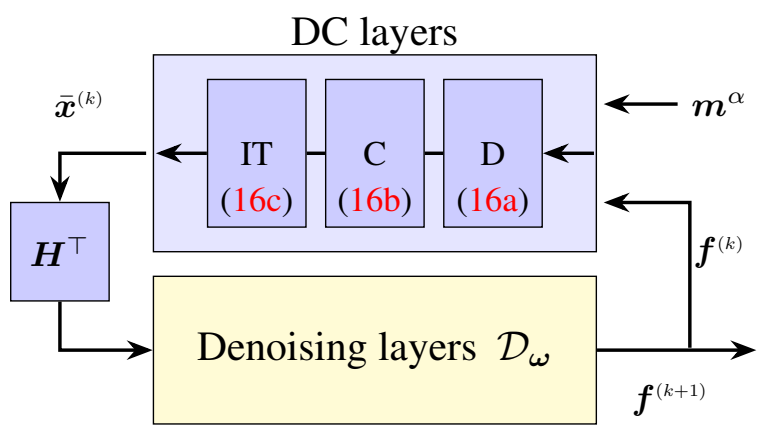

Fig. 1. Proposed deep EM reconstruction network. The data consistency (DC) layers consist of three steps: the denoising (D) step, the completion (C) step, and the inverse transform (IT) step. The denoising layers $\mathcal{D}_{\boldsymbol{\omega}}$ implement a U-Net [20].

where $\boldsymbol{\Sigma}_{1} \in \mathbb{R}^{M \times M}, \boldsymbol{\Sigma}_{21} \in \mathbb{R}^{(N-M) \times M}$ and $\boldsymbol{\Sigma}_{2} \in$ $\mathbb{R}^{(N-M) \times(N-M)}$ are the blocks of the covariance $\boldsymbol{\Sigma}$

$$
\boldsymbol{\Sigma}=\left[\begin{array}{cc}
\boldsymbol{\Sigma}_{1} & \boldsymbol{\Sigma}_{21}^{\top} \\
\boldsymbol{\Sigma}_{21} & \boldsymbol{\Sigma}_{2}
\end{array}\right]
$$

To circumvent the difficulty of inverting the signal-dependant matrix in Equation (13), we choose to neglect the nondiagonal terms of $\boldsymbol{\Sigma}_{1}$, as in [19]. Denoting $\boldsymbol{\sigma}_{1}^{2}=\operatorname{diag}\left(\boldsymbol{\Sigma}_{1}\right)$, we get

$$
\boldsymbol{y}_{1}^{(k)}\left(\boldsymbol{m}^{\alpha}\right)=\boldsymbol{\sigma}_{1}^{2} /\left(\boldsymbol{\sigma}_{1}^{2}+\boldsymbol{\sigma}_{\alpha}^{2}\right)\left(\boldsymbol{m}^{\alpha}-\boldsymbol{H}_{1} \boldsymbol{f}^{(k)}\right),
$$

where division and multiplication apply element-wise.

Finally, we can summarize our algorithm by

$$
\begin{aligned}
\boldsymbol{y}_{1}^{(k)} & =\boldsymbol{\sigma}_{1}^{2} /\left(\boldsymbol{\sigma}_{1}^{2}+\boldsymbol{\sigma}_{\alpha}^{2}\right)\left(\boldsymbol{m}^{\alpha}-\boldsymbol{H}_{1} \boldsymbol{f}^{(k)}\right) \\
\boldsymbol{y}_{2}^{(k)} & =\boldsymbol{\Sigma}_{21} \boldsymbol{\Sigma}_{1}^{-1} \boldsymbol{y}_{1}^{(k)} \\
\overline{\boldsymbol{x}}^{(k)} & =\boldsymbol{H} \boldsymbol{f}^{(k)}+\boldsymbol{y}^{(k)} \\
\boldsymbol{f}^{(k+1)} & =\mathcal{D}_{\boldsymbol{\omega}}\left(\boldsymbol{H}^{\top} \overline{\boldsymbol{x}}^{(k)}\right) .
\end{aligned}
$$

The outline of our algorithm can be found in Fig. 1. In this scheme, we propose to learn $\sigma_{1}, \Sigma_{21} \boldsymbol{\Sigma}_{1}^{-1}$, and $\mathcal{D}_{\boldsymbol{\omega}}$ in an endto-end fashion. Note that as in [10], $\boldsymbol{\sigma}_{1}, \boldsymbol{\Sigma}_{21} \boldsymbol{\Sigma}_{1}^{-1}$, and the parameters of our network $\mathcal{D}_{\boldsymbol{\omega}}$ are shared across every iteration $0 \leq k \leq K$ to reduce the number of learned parameters.

\subsection{Denoised completion in the presence of Poisson noise}

By using the normal approximation to the Poisson distribution (see [21]), Equation (1) can be approximated as

$$
\mathbf{m}^{\alpha} \sim \frac{1}{\alpha}\left[\mathcal{N}\left(\alpha \boldsymbol{H}_{1}^{+} \boldsymbol{f}, \hat{\boldsymbol{\Sigma}}_{\alpha}^{+}\right)-\mathcal{N}\left(\alpha \boldsymbol{H}_{1}^{-} \boldsymbol{f}, \hat{\boldsymbol{\Sigma}}_{\alpha}^{-}\right)\right],
$$

where $\hat{\boldsymbol{\Sigma}}_{\alpha}^{+}=\operatorname{Diag}\left(\alpha \boldsymbol{H}_{1}^{+} \boldsymbol{f}\right)$ and $\hat{\boldsymbol{\Sigma}}_{\alpha}^{-}=\operatorname{Diag}\left(\alpha \boldsymbol{H}_{1}^{-} \boldsymbol{f}\right)$. This can also be expressed as

$$
\boldsymbol{\Sigma}_{\alpha}=\operatorname{Diag}\left(\boldsymbol{\sigma}_{\alpha}^{2}\right)=\operatorname{Diag}\left(\frac{1}{\alpha} \boldsymbol{H}_{1}^{+} \boldsymbol{f}+\frac{1}{\alpha} \boldsymbol{H}_{1}^{-} \boldsymbol{f}\right) .
$$
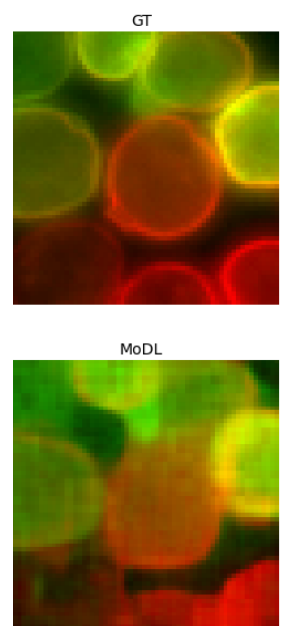
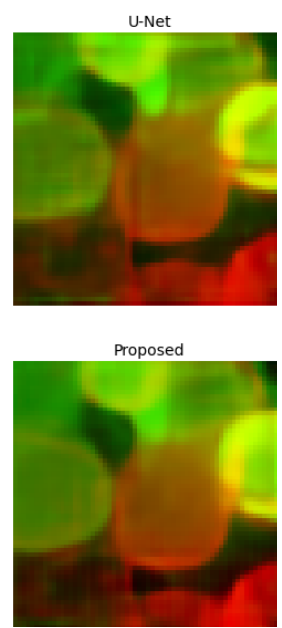

Fig. 2. Reconstruction of a yeast cell membrane fluorescence microscopy image.

As $\boldsymbol{\sigma}_{\alpha}^{2}$ depends on the unknown image $\boldsymbol{f}$ as well as on the intensity $\alpha$, we exploit the raw data that also depends on $\boldsymbol{f}$ and $\alpha$. Recalling that the variance of a Poisson variable is the same as its expected value, we can write $\hat{\boldsymbol{\Sigma}}_{\alpha}^{+}=\mathbb{E}\left(\hat{\mathbf{m}}_{+}^{\alpha}\right)$ and $\hat{\boldsymbol{\Sigma}}_{\alpha}^{-}=\mathbb{E}\left(\hat{\mathbf{m}}_{-}^{\alpha}\right)$, and choose to approximate the expected value by the noisy sample, i.e., $\boldsymbol{\sigma}_{\alpha}^{2} \approx 1 / \alpha^{2}\left(\hat{\boldsymbol{m}}_{+}^{\alpha}+\hat{\boldsymbol{m}}_{-}^{\alpha}\right)$.

\section{EXPERIMENTS}

In our experiments, we chose $M=512$ Hadamard patterns of size $N=64 \times 64$ pixels. We trained our network for $K=5$ iterations in a end-to-end fashion. The architecture of $\mathcal{D}_{\boldsymbol{\omega}}$ is a U-net [20]. We consider two network variants: (i) a standalone (direct) U-net reconstructor, which is equivalent to one iteration of our algorithm; and (ii) model-based reconstruction using deep-learned priors (MoDL) [10], which is equivalent to setting $\boldsymbol{\Sigma}_{21}=\mathbf{0}$ and $\boldsymbol{\Sigma}_{1}=\lambda \boldsymbol{I}$ in Equation (16). All of the networks are trained using 105,000 images (i.e., the 'unlabeled' and 'train' subsets of the STL10 database [22]); 8,000 images were used for the test (i.e., the 'test' subset of STL10). The original $96 \times 96$ images were cropped to $64 \times 64$, and normalized between -1 and 1 . We train our unrolled network using Pytorch [23]. For training, we consider the ADAM optimiser for 20 epochs. The step size is initialized to $10^{-3}$ and divided by 5 every 5 epochs. The weight decay regularization parameter is set to $10^{-6}$. The number of learned parameters is $4,432,657$. Note that our initial estimate of $\boldsymbol{f}^{(0)}$ is computed as described in [24].

\section{RESULTS AND DISCUSSION}

In Table 1, we compare our proposed deep EM network with three other methods: the Moore-Penrose pseudo inverse (PI), a stand-alone U-net, and MoDL [10]. According to our sim- 


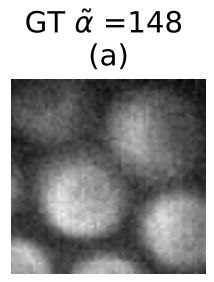

GT $\tilde{\alpha}=195$

(b)

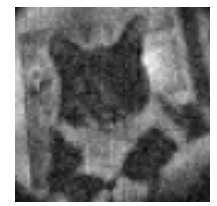

GT $\tilde{\alpha}=80$

PSNR $=24.07$

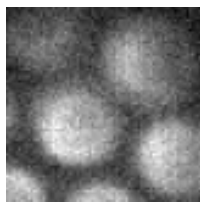

GT $\tilde{\alpha}=10$

PSNR $=13.46$

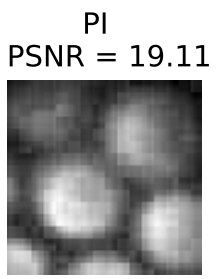

PI

PSNR $=16.23$
U-Net

PSNR $=22.8$

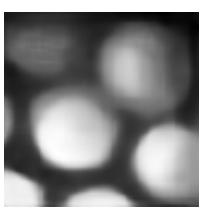

U-Net

PSNR $=18.99$
MoDL

PSNR $=22.1$

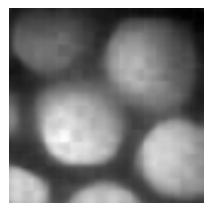

MoDL

PSNR $=18.1$

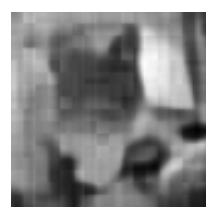

Proposed

PSNR $=23.18$

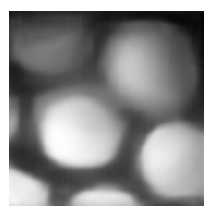

Proposed PSNR $=19.66$

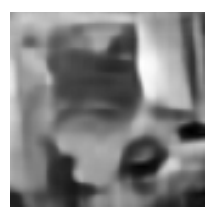

Fig. 3. Reconstructions of two experimental datasets by the different methods (top row: LED lamp with $M=512$; bottom row: STL-10 cat with $M=512$ ). The images shown were reconstructed from a fully sampled dataset (ground-truth; GT) acquired with high image intensity (first column, $\alpha=148$ photons, and $\alpha=195$ photons) and lower image intensity (second column, $\alpha=80$ photons, and $\alpha=10$ photons). The following columns show reconstructions using pseudo inverse (PI), U-Net reconstructor, MoDL [10], and the proposed method.

ulations, our method outperforms the other methods in terms of peak signal-to-noise ratio (PSNR) and structural similarity (SSIM) for different image intensities $\alpha$ (i.e., different levels of noise). Note that the benefits of our method are more apparent for high noise (i.e., low $\alpha$ ).

In Fig. 2, we consider fluorescence microscopy imaging of yeast cell membranes, which do not belong to the STL10 test set. The membrane proteins are fused with red and green fluorescent protein markers. Each channel was treated independently. We obtain an improved PSNR in the red channel with the proposed network $(23.14 \mathrm{~dB}$ for the proposed method, compared to $22.99 \mathrm{~dB}$ for U-net, and $22.03 \mathrm{~dB}$ for MoDL). For the green channel, however, MoDL [10] gives the best PSNR $(19.33 \mathrm{~dB})$ followed by our proposed method $(17.92 \mathrm{db})$ and then U-net (17.04 dB). Visually, our deep EM reconstruction presents less strike artefacts than the alternative methods.

In Fig. 3, we finally assess our deep EM network using experimental data obtained using the set-up described in [19]. We obtain improved PSNR with the proposed method. Visually, the deep EM network and the stand-alone U-net yield images with fewer compression artifacts. The MoDL [10] reconstruction is noisier, which can be explained on the basis that each iteration assumes signal-independent Gaussian noise, rather than signal-dependent noise.

\section{CONCLUSION AND PERSPECTIVES}

We propose a recursive network based on the EM algorithm. This deep EM network can solve linear underdetermined inverse problems where the data are corrupted by signal-dependent noise (e.g., Poisson noise, mixed Poisson-
Gaussian noise). This approach is shown to yield greater PSNR under several levels of noise compared to the the U-net approach alone or a MoDL type of approach. To the best of our knowledge, most unrolled networks assume noise to be Gaussian with constant variance, and are therefore ill-suited for compressive optics applications. The proposed method is promising for single-pixel biological imaging and can further be adapted to the reconstruction of other types of biophotonic imaging modalities.

\begin{tabular}{lccccc}
\hline$\alpha($ ph. $)$ & & PI & U-Net & MoDL & Proposed \\
\hline \multirow{2}{*}{2} & PSNR & 12.97 & 17.17 & 17.14 & $\mathbf{1 7 . 8 2}$ \\
& SSIM & 0.61 & 0.72 & 0.73 & $\mathbf{0 . 7 8}$ \\
\hline \multirow{2}{*}{5} & PSNR & 15.32 & 19.41 & 19.49 & $\mathbf{1 9 . 8 5}$ \\
& SSIM & 0.73 & 0.81 & 0.81 & $\mathbf{0 . 8 5}$ \\
\hline \multirow{2}{*}{10} & PSNR & 16.51 & 20.61 & 20.7 & $\mathbf{2 0 . 8 8}$ \\
& SSIM & 0.78 & 0.85 & 0.84 & $\mathbf{0 . 8 8}$ \\
\hline \multirow{2}{*}{50} & PSNR & 17.8 & 21.8 & 21.42 & $\mathbf{2 1 . 8 9}$ \\
& SSIM & 0.82 & 0.9 & 0.85 & $\mathbf{0 . 9}$ \\
\hline \multirow{2}{*}{2500} & PSNR & 18.17 & 22.11 & 21.55 & $\mathbf{2 2 . 1 5}$ \\
& SSIM & 0.84 & 0.91 & 0.86 & $\mathbf{0 . 9 1}$ \\
\hline
\end{tabular}

Table 1. Average peak signal-to-noise ratio (PSNR) and structural similarity (SSIM) over the stl-10 test dataset for the different reconstruction methods: PI (pseudo inverse), standalone U-Net, MoDL, and the proposed deep EM network.

\section{COMPLIANCE WITH ETHICAL STANDARDS}

This paper reports no data from studies involving human and/or animal subject. 


\section{ACKNOWLEDGMENTS}

This work was supported by the French National Research Agency (ANR), under Grant ANR-17-CE19-0003 (ARMONI Project), and performed within the framework of the LABEX PRIMES (ANR-11-LABX-0063) of Université de Lyon.

\section{REFERENCES}

[1] Matthew P. Edgar et al., "Principles and prospects for single-pixel imaging," Nature Photonics, vol. 13, no. 1, pp. 13-20, Jan. 2019.

[2] M.F. Duarte et al., "Single-pixel imaging via compressive sampling," Signal Processing Magazine, IEEE, vol. 25, no. 2, pp. 83-91, March 2008.

[3] Vincent Studer et al., "Compressive fluorescence microscopy for biological and hyperspectral imaging," Proceedings of the National Academy of Sciences, vol. 109, no. 26, pp. E1679-E1687, 2012.

[4] Florian Rousset et al., "Time-resolved multispectral imaging based on an adaptive single-pixel camera," Opt. Express, vol. 26, no. 8, pp. 10550-10558, Apr 2018.

[5] Enagnon Aguénounon et al., "Single snapshot imaging of optical properties using a single-pixel camera: a simulation study," Journal of Biomedical Optics, vol. 24, no. 7, pp. $1-6,2019$.

[6] Ruoyang Yao et al., "Net-flics: fast quantitative widefield fluorescence lifetime imaging with compressed sensing - a deep learning approach," Light: Science \& Applications, vol. 8, no. 1, pp. 26, Mar. 2019.

[7] Simon Arridge et al., "Solving inverse problems using data-driven models," Acta Numerica, vol. 28, pp. 1-174, 2019.

[8] M. T. McCann et al., "Convolutional neural networks for inverse problems in imaging: A review," IEEE Signal Processing Magazine, vol. 34, no. 6, pp. 85-95, 2017.

[9] Eunhee Kang et al., "Cycle-consistent adversarial denoising network for multiphase coronary $\mathrm{CT}$ angiography," Medical Physics, vol. 46, no. 2, pp. 550-562, Dec. 2018.

[10] H. K. Aggarwal et al., "Modl: Model-based deep learning architecture for inverse problems," IEEE Transactions on Medical Imaging, vol. 38, no. 2, pp. 394-405, 2019.

[11] H. Gupta et al., "Cnn-based projected gradient descent for consistent ct image reconstruction," IEEE Transactions on Medical Imaging, vol. 37, no. $\overline{6, \mathrm{pp}}$. 1440-1453, June 2018.
[12] J. A. Fessler et al., "Space-alternating generalized expectation-maximization algorithm," IEEE Transactions on Signal Processing, vol. 42, no. 10, pp. 2664-2677, 1994.

[13] M. Ochoa et al., "Assessing patterns for compressive fluorescence lifetime imaging," Opt. Lett., vol. 43, no. 18, pp. 4370-4373, Sep 2018.

[14] A. Foi et al., "Practical poissonian-gaussian noise modeling and fitting for single-image raw-data," IEEE Transactions on Image Processing, vol. 17, no. 10, pp. 1737-1754, Oct. 2008.

[15] Antonio Lorente Mur et al., "Handling negative patterns for fast single-pixel lifetime imaging," in SPIE Photonics : Molecular-Guided Surgery: Molecules, Devices, and Applications V, 2019, vol. 10862.

[16] G. Ongie et al., "Deep learning techniques for inverse problems in imaging," IEEE Journal on Selected Areas in Information Theory, vol. 1, no. 1, pp. 39-56, May 2020.

[17] J. Zhou et al., "A bayesian map-em algorithm for pet image reconstruction using wavelet transform," IEEE Transactions on Nuclear Science, vol. 54, no. 5, pp. 1660-1669, 2007.

[18] Albert Tarantola, Inverse Problem Theory and Methods for Model Parameter Estimation, Society for Industrial and Applied Mathematics, 2005.

[19] Antonio Lorente Mur et al., "A Deep Network for Reconstructing Images from Undersampled Poisson data," working paper or preprint, Sept. 2020.

[20] Olaf Ronneberger et al., "U-net: Convolutional networks for biomedical image segmentation," CoRR, vol. abs/1505.04597, 2015.

[21] Kerson Huang, Introduction to statistical physics, Taylor \& Francis, London New York, 2001.

[22] Adam Coates et al., "An analysis of single-layer networks in unsupervised feature learning," in Proceedings of the fourteenth international conference on artificial intelligence and statistics, 2011, pp. 215-223.

[23] Adam Paszke et al., "Pytorch: An imperative style, high-performance deep learning library," in Advances in Neural Information Processing Systems 32, pp. 80248035. Curran Associates, Inc., 2019.

[24] N. Ducros et al., "A completion network for reconstruction from compressed acquisition," in 2020 IEEE 17th International Symposium on Biomedical Imaging (ISBI), 2020, pp. 619-623. 